Portland State University

PDXScholar

$5-1-1988$

\title{
Spectral behavior and pulse train instabilities of a synchronously pumped mode-locked dye laser
}

\author{
Duncan Leo MacFarlane \\ Lee W. Casperson \\ Portland State University \\ Anthony A. Tovar
}

Follow this and additional works at: https://pdxscholar.library.pdx.edu/ece_fac

Part of the Electrical and Computer Engineering Commons

Let us know how access to this document benefits you.

\section{Citation Details}

D. L. MacFarlane, Lee W. Casperson, and A. A. Tovar, "Spectral behavior and pulse train instabilities of a synchronously pumped mode-locked dye laser," J. Opt. Soc. Am. B 5, 1144-1152 (1988).

This Article is brought to you for free and open access. It has been accepted for inclusion in Electrical and Computer Engineering Faculty Publications and Presentations by an authorized administrator of PDXScholar. Please contact us if we can make this document more accessible: pdxscholar@pdx.edu. 


\title{
Spectral behavior and pulse train instabilities of a synchronously pumped mode-locked dye laser
}

\author{
D. L. MacFarlane, Lee W. Casperson, and A. A. Tovar \\ Department of Electrical Engineering, Portland State University, Portland, Oregon 97207
}

Received September 15, 1987; accepted December 2, 1987

\begin{abstract}
The pulse-train envelope of a synchronously pumped mode-locked dye laser has been experimentally studied as a function of cavity length detuning. When a bandwidth-limiting birefringent tuning filter is included and the cavity is adjusted to the length that yields optimally short $(\sim 2$-psec $)$ pulses, there is a slow $(\leq 800 \mathrm{kHz})$, erratic amplitude modulation that provides a $20 \%$ variation in pulse energies. As the cavity length is detuned on the order of one part in $10^{4}$, the modulation vanishes, but the individual pulse lengths increase dramatically. Further detuning results in a clean periodic envelope with a $20 \%$ modulation depth and a 5- $\mu$ sec period. With the birefringent filter removed, the dye-laser wavelength and bandwidth are studied as functions of the cavity length. Temporally, the clean periodic modulation persists at large detunings, but there is no length at which any erratic modulation can be observed without the bandwidth-limiting filter.
\end{abstract}

\section{INTRODUCTION}

Since the first reports of active mode locking in lasers, ${ }^{1}$ much demand has arisen for the stable, ultrashort optical pulses produced by this technique. Consequently much effort has gone into the theory and practical realization of reliable mode-locked pulse trains that are pulse-to-pulse consistent. A class of considerable interest is the synchronously pumped mode-locked (SPML) dye laser, which currently finds wide use as a tunable source of picosecond and even subpicosecond optical pulses. ${ }^{2}$ A typical system employs a cw modelocked pump laser, such as an acousto-optically modelocked argon-ion laser whose cavity length is almost equal to the cavity length of the dye laser. Since the earliest experimental reports, ${ }^{3,4}$ this arrangement has been refined into a turnkey commercial system.

The earliest theoretical studies of SPML lasers used a rate-equation analysis to study steady-state pulse parameters as functions of the length mismatch between the pump laser cavity and that of the dye laser. ${ }^{5,6}$ Subsequent papers augmented these treatments, but it is only recently that theories have questioned the steady-state or self-consistent pulse-profile approach. ${ }^{7-10}$ Experimentally, Kluge et al..$^{11}$ studied the subpicosecond temporal jitter on the output train of a SPML dye laser. They attribute this temporal jitter to the jitter of the pump laser. The theoretical studies of New and Catherall, however, suggested that a SPML laser will have a fundamental source of jitter. ${ }^{9}$ Further, streakcamera studies have suggested that there are cavity-length regimes-particularly where the shortest mode-locked pulses occur-where SPML dye lasers are unstable. ${ }^{12}$ Separately, Baer and Smith reported on the comb-structured rf spectra of a slightly mismatched SPML dye laser. ${ }^{13}$

This paper presents the results of a systematic experimental study of the effect of small cavity detunings on the pulsetrain envelope of a SPML Rhodamine $6 \mathrm{G}$ dye laser. Although the behavior of the laser is rich and varied, of practical interest is the observation of a slow, erratic amplitude modulation at the cavity length that produces the shortest pulses when the bandwidth-limiting tuning filter is included in the dye-laser cavity. Because most theories governing SPML dye lasers employ some form of a self-consistent pulse approximation, the behavior reported here has not been predicted. However, significant variations (up to 20\%) occur only over the course of hundreds of pulses. Thus actual pulse-to-pulse variations are small, and the self-consistent pulse approximation remains reasonable for many purposes.

Zheng et al. reported a sequence of period-doubling bifurcations associated with extreme (30\%) length mismatches between a mode-locked argon-ion pump laser and a Rhodamine dye laser and suggest that this system is appropriate for studies of optical chaos. ${ }^{14}$ The range of cavity detunings explored here, however, is kept to within 1 part in $1000-a$ physical length shift of $1.4 \mathrm{~mm}$. This detuning range and the observed $\sim 10^{5}-\mathrm{Hz}$ amplitude modulation is parallel to a recent study of an acousto-optically mode-locked argon-ion laser. ${ }^{15}$ Reference 15 and the results of this exploration suggest that a slow, erratic envelope modulation may be a general characteristic of slightly detuned, actively modelocked laser systems. An understanding of this instability may lead to a more thoughtful design of mode-locking drivers.

Similar time-domain studies have also been carried out without the filter in the cavity. Interestingly, no erratic behavior of the pulse-train envelope is observed when this linear element is omitted. However, a slow (microsecond) periodic modulation of the pulse train is identically present with and without the filter. Unlike the periodic modulation reported in Ref. 15, there is no onset of erratic behavior on further detuning. For the dye laser there is only a linear increase in fundamental modulation frequency with detuning.

There has recently been considerable interest in the bichromatic spectra emitted by cw dye lasers at suitable values of the threshold parameter $r .{ }^{16,17}$ In this study we also 
examine the spectroscopy of a SPML dye laser as a function of the cavity length detuning, and certain types of multiwavelength emissions are also reported.

\section{EXPERIMENTAL SETUP}

The experiments were performed using a commercially available Spectra-Physics argon-ion/Rhodamine 6G dye-laser system with cavity lengths of $186 \mathrm{~cm}$. the argon laser (Spectra-Physics 2020) was actively mode locked by a fused silica acousto-optic modulator driven at about $40.25 \mathrm{MHz}$. The argon laser was operated in its $\mathrm{TEM}_{00}$ transverse mode and was set up to produce a stable train of 100-psec, 514-nm pulses with a pulse repetition frequency (PRF) of $80.5 \mathrm{MHz}$. Any jitter on this pulse train was more than $60 \mathrm{~dB}$ below the level of the 80.5 PRF component.

The argon laser pumped a matched cavity length SpectraPhysics 375B dye laser at a level approximately 2.4 times above threshold. The details of this arrangement are presented in Fig. 1. The $2 \times 10^{-3} \mathrm{M}$ (Rhodamine 6G in ethylene glycol) dye jet was set at Brewster's angle to the argon laser beam. The output coupler was mounted on a translatable stage, which provided the cavity-length adjustment. A Mitutoyo dial indicator permitted displacement measurements readable to $0.5-\mu \mathrm{m}$ resolution, and the relative system-length error was less than $\pm 2 \mu \mathrm{m}$. For our purposes the optimum cavity length was defined as that length giving the shortest mode-locked pulse, as measured by a Spectra-Physics 409 autocorrelator. Length detunings were then measured as displacements away from this position. A reasonable estimate of this optimum difference between the length of the argon laser and the dye laser can be garnered from Ref. 18 and, for this case, is approximately $50 \mu \mathrm{m}$.

The temporal character of the pulse train was studied using two fast Si P-I-N diodes (Antronics S-2) operating photoconductively. The entire cross section of the laser beam was focused onto the detectors, although no variations

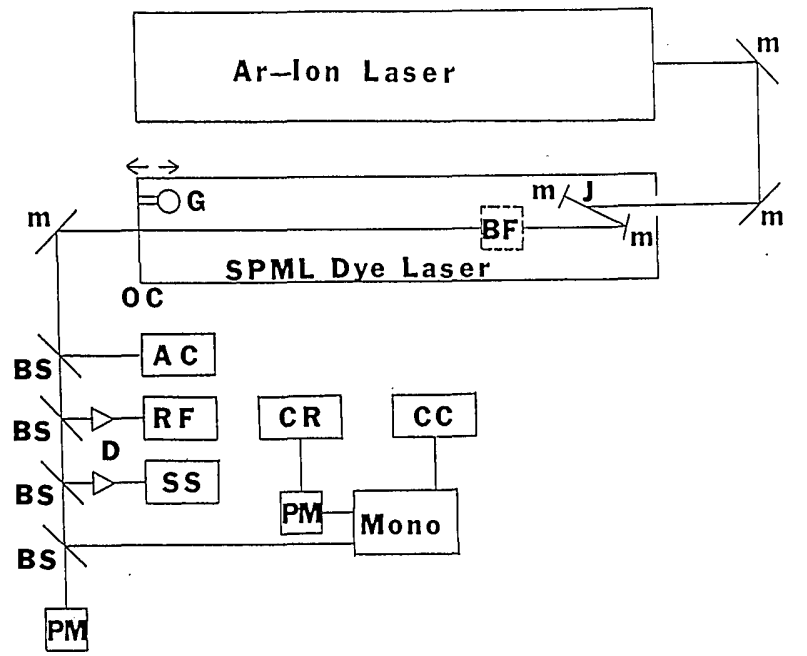

Fig. 1. Schematic representation of the experimental setup for the study of the spectra and the pulse train from an SPML dye laser: $\mathrm{AC}$, autocorrelator; BF, birefringent filter; BS's, beam splitters; CC, computer controller; CR, chart recorder; D, detector; $\mathrm{G}$, length measuring gauge; J, dye jet; M's, mirrors; Mono, monochromator; OC, output coupler; PM's, power meters; RF, RF spectrum analyzer; SS, real-time storage scope. in the effects reported here were observed when the detector was scanned radially across the beam. Although the 35-psec rise time of these detectors prevented any study of individual pulse shape, their signals provided an adequate measure of the total energy in each pulse. One of the signals was fed to a Tektronix 7L12 rf spectrum analyzer in a 7904 mainframe. This setup permitted constant monitoring of the homodyne spectrum as a function of length detuning. In general, it was convenient to focus on the frequency range of $\pm 1 \mathrm{MHz}$ around the $80.5-\mathrm{MHz}, c / 2 L, \mathrm{PRF}$. The second photodiode was used to study the envelope in the time domain by using a Tektronix 7834 storage scope. The storage mode was essential for viewing some of the irregular (nontriggerable) signals. Also monitored was the average output power as measured by a Coherent 212 power meter. Interestingly, the average power remained fairly constant at approximately $75 \mathrm{~mW}( \pm 10 \%)$ throughout the range of detunings.

Generally included in the cavity was a two-plate birefringent filter with a measured bandwidth of $12 \mathrm{~nm}$ (FWHM). This permitted 2-psec pulses that were tunable across the Rhodamine gain spectrum. When the birefringent filter was removed, the operating wavelength and the bandwidth of the dye laser were no longer predetermined, and the resulting spectroscopy of the laser became interesting. The wavelength dependence of the average power was studied using a motor-driven 0.5-m Jarrell-Ash monochromator. A Coherent 212 power meter measured the average power transmitted by the monochromator. This analog signal was recorded on a Hewlett-Packard 7047A $x-y$ recorder.

\section{TIME AND RADIO-FREQUENCY SPECTRA DATA}

Of immediate practical interest is the observation of a slow, erratic modulation on the pulse train at the optimum length detuning with the inclusion of a bandwidth-limiting, wavelength-tuning birefringent filter. Much of the data in the following paragraphs was taken with the birefringent filter in place and tuned to the peak wavelength of $599 \mathrm{~nm}$; however, the data are qualitatively similar at all wavelengths across the Rhodamine gain curve. Figure 2(a) is a storagescope trace of the pulse-train envelope at the optimum cavity length. On the $2 \mu \mathrm{sec} /$ division scale shown here, the individual pulses are crowded together and the envelope appears as a continuous trace, showing significant modulation. The frequency content of this envelope is shown in the rf spectrum analyzer trace of Fig. 2(b). The horizontal scale is $200 \mathrm{kHz} /$ division. The central peak is the $80.5-\mathrm{MHz}$, $c / 2 L, \mathrm{PRF}$ and is surrounded by a broad background that lifts off the baseline. This homodyne spectrum reflects an irregular amplitude modulation of the pulse train with frequency components out to $800 \mathrm{kHz}$.

Pulses of under 10-psec duration are produced only if the cavity length is between $L_{\mathrm{opt}}-10 \mu \mathrm{m}$ and $L_{\mathrm{opt}}+30 \mu \mathrm{m}$. The irregular envelope shown in Fig. 2(a) is present throughout this $40-\mu \mathrm{m}$ range of lengths. The modulation depth, however, does decrease as the pulse length increases. At $L=L_{\text {opt }}$ $+35 \mu \mathrm{m}$, the modulation is $60 \mathrm{~dB}$ down, while the pulse widths are greater than 30 psec. Further detuning to $L=$ $L_{\text {opt }}+92 \mu$ m produces a deep (25\%) regular envelope modulation. This clean periodic modulation persists for further 
length increases until lasing stops at $L=L_{\text {opt }}+950 \mu \mathrm{m}$. Figure 3 shows temporal traces and homodyne spectra for $L$ $=L_{\mathrm{opt}}+125 \mu \mathrm{m}$ and $L=L_{\mathrm{opt}}+320 \mu \mathrm{m}$. As the cavity length increases through this regime, the modulation frequency increases linearly with a slope of $1.09 \mathrm{kHz} / \mu \mathrm{m}$, as shown in Fig. 4. Although this periodic modulation sets in with an abrupt threshold at a detuning of $+92 \mu \mathrm{m}$, if one projects a best-fit line through a plot of modulation frequency versus detuning, one finds a modulation frequency of zero at a detuning of $+3 \mu \mathrm{m}$-effectively at the optimum length.

The phenomenology is more varied when $L<L_{\text {opt }}$. The broadband noise present at $L_{\text {opt }}$ persists as $L$ is initially shortened. At first the bandwidth of the envelope narrows and the modulation depth gradually decreases. At $L=L_{\mathrm{opt}}$ $-60 \mu \mathrm{m}$, there is a sharp transition point at which several strong sidebands grow from the background. In the time domain this appears as a sudden increase in the modulation depth. The pulse-train envelope and its homodyne spectrum at this length are shown in Fig. 5. This modulation diminishes on further detuning until, at $L=L_{\mathrm{opt}}-80 \mu \mathrm{m}$, the envelope is essentially flat. A second sharp transition into modulation occurs at $L=L_{\mathrm{opt}}-123 \mu \mathrm{m}$, at which point a strong, noisy modulation develops, as shown in Fig. 6. The irregularity in this signal disappears and the modulation depth grows during the next $5-\mu \mathrm{m}$ length decrease. This case is shown in Fig. 7 for $L=L_{\text {opt }}-128$. The power spectrum of Fig. 7(b) suggests that a period-5 bifurcation has occurred since $L=L_{\mathrm{opt}}-123 \mu \mathrm{m}$. Over the next $100 \mu \mathrm{m}$ these smaller components grow, and frequency components fill in between them. In the time domain there is an irregular envelope with a $50 \%$ modulation depth. Lasing stops at $L=L_{\text {opt }}-280 \mu \mathrm{m}$.

The data described above were taken with the birefringent filter in the dye-laser cavity. The pulse-train phenomenology is markedly simpler when this element is removed. In particular, there is no length regime where the envelope of the pulse train may be called erratic, including near $L_{\text {opt }}$. Although there are areas for $L<L_{\text {opt }}$ where there is a measurable background on the rf spectrum, its level is always down by more than $60 \mathrm{~dB}$ from the $80.5-\mathrm{MHz}$ PRF component.

A characteristic that identically occurs whether or not the

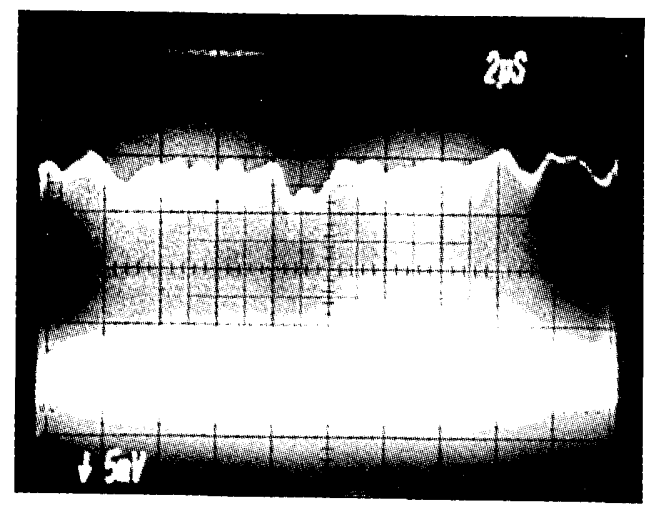

(a)

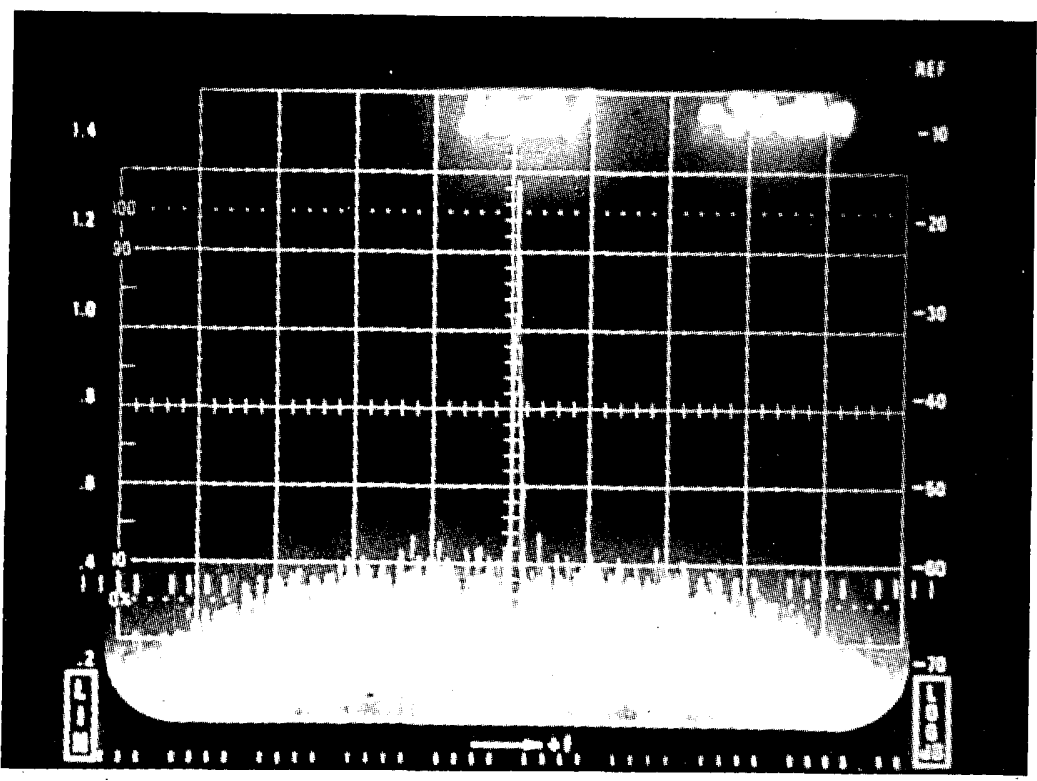

(b)

Fig. 2. (a) Temporal and (b) rf homodyne spectral representations of the pulse-train envelope at $L_{\text {opt. }}$ The horizontal scale in (a) is $2 \mu$ sec/division. In (b) the scales are $200 \mathrm{kHz} /$ division and $10 \mathrm{~dB} /$ division. The spectra are centered about the $80.5-\mathrm{MHz}, c / 2 L$ component. 


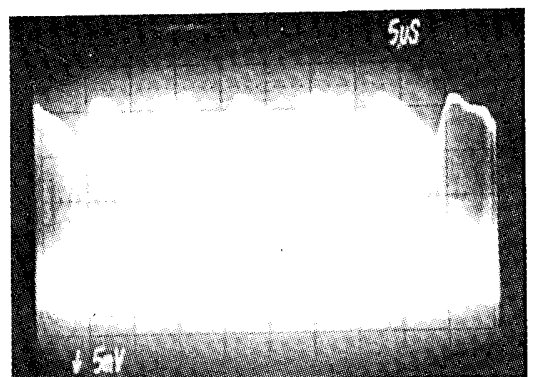

(a)

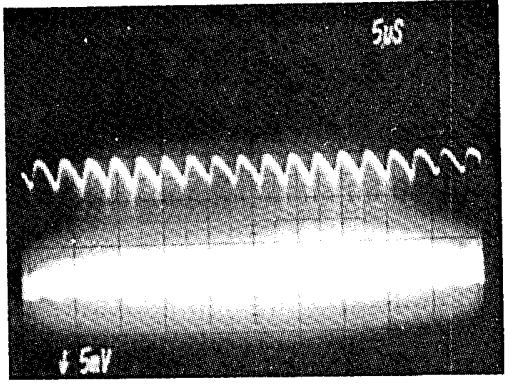

(c)

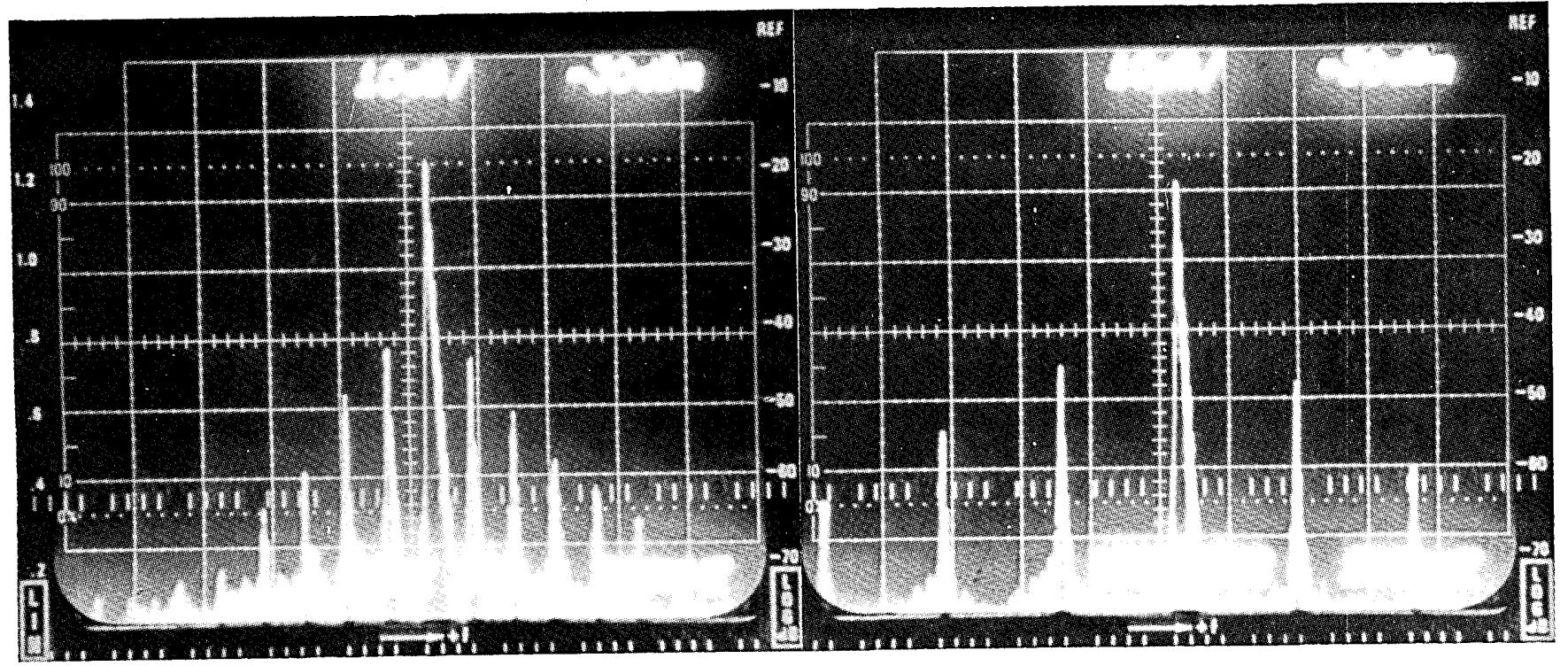

(b)

(d)

Fig. 3. Clean periodic envelope modulation at long cavity lengths. For (a) and (b), $L=L_{\mathrm{opt}}+125 \mu \mathrm{m}$; for (c) and (d), $L=L_{\mathrm{opt}}+320 \mu \mathrm{m}$. The time base of (a) and (c) is $5 \mu \mathrm{sec} /$ division. The spectrum analyzer scales in (b) and (d) are the same as in Fig. 2.

tuning filter is in the cavity, however, is the clean periodic envelope modulation, as in Fig. 3. Temporal data taken without the filter give the same sudden onset at $L_{\mathrm{opt}}+92 \mu \mathrm{m}$ and the same linear shift in frequency on further detuning, as shown in Fig. 4.

\section{SPECTRAL BEHAVIOR OF A FREE-RUNNING SYNCHRONOUSLY PUMPED MODE-LOCKED DYE LASER}

When the dye laser is free running, that is to say, when there is no wavelength-determining intracavity filter, the peak lasing wavelength, the bandwidth, and the shape of the spectra all become functions of the cavity length. The fact that the lasing wavelength changes with cavity length is not an anticipated result and does not occur in cw dye lasers. In Fig. 8 is a plot of lasing wavelength versus cavity detuning. Immediately evident are striking transitions in $\lambda_{\text {peak }}$ at $\Delta L=$ $-437 \mu \mathrm{m}$ and $\Delta L=+92 \mu \mathrm{m}$. These transitions represent points at which the lasing wavelength is extremely sensitive to cavity length. No hysteresis was observed beyond that attributed to the mechanical setup. It is noteworthy that the transition at $\Delta L=+92 \mu \mathrm{m}$ corresponds to the threshold detuning at which the uniform pulse train develops a period-

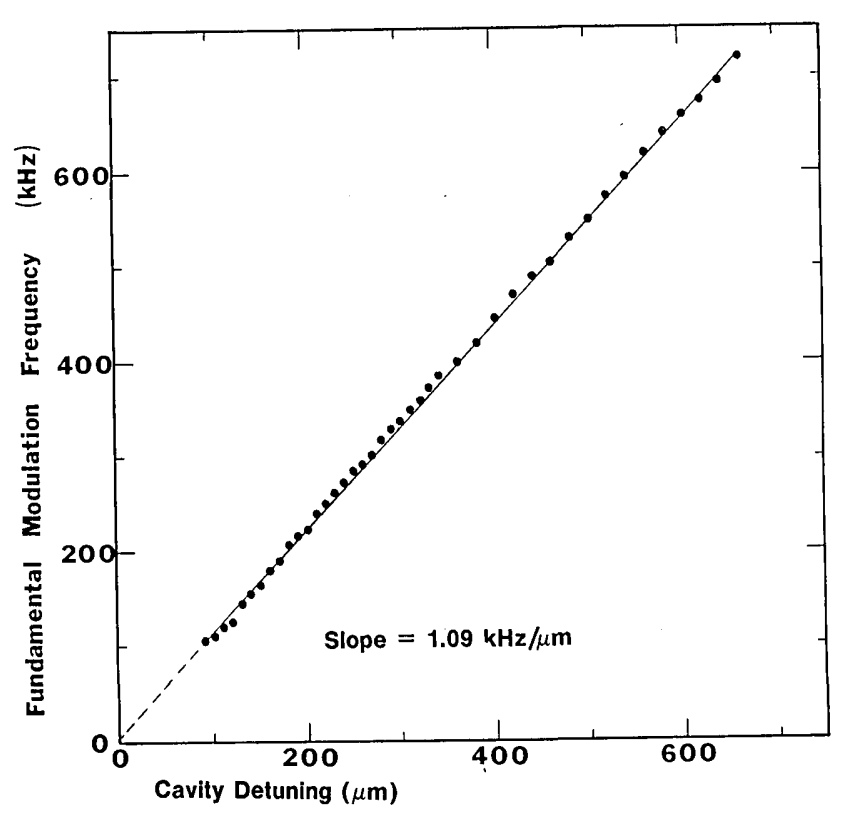

Fig. 4. Pulse-train modulation frequency versus cavity length detuning and a linear best fit. Typical error bars are of the order of the spot diameter. 
ic modulation. Also of interest is the region of trichromatic emission between $L=L_{\text {opt }}+540 \mu \mathrm{m}$ and $L=L_{\text {opt }}+620 \mu \mathrm{m}$.

The structure of the emission spectra also changes significantly with cavity-length detuning, and Fig. 9 presents a sampling of typical spectra in an abbreviated sequence for (a) $L=L_{\text {opt }}-35 \mu \mathrm{m}$, (b) $L=L_{\text {opt }}$, (c) $L=L_{\text {opt }}+92 \mu \mathrm{m}$ (the threshold for the periodic modulation), and (d) $L=L_{\mathrm{opt}}+$ $280 \mu \mathrm{m}$. Through the transitions noted above, the average power out of the dye laser does not change appreciably. In fact, as a rule, the average power is a fairly smooth function of detuning that peaks around $L=L_{\text {opt }}+50 \mu \mathrm{m}$. Consequently, although the bandwidth and the peak heights of these spectra change (at times abruptly and drastically), the area under each of these curves is roughly constant.

As the cavity is further lengthened, the main peak of Fig. 9(d) diminishes, and the shoulder of Fig. 9(c) returns. The resulting spectra are exemplified in Fig. 10 for $L=L_{\text {opt }}+585$ $\mu \mathrm{m}$, which may be viewed as an example of trichromatic emission. The separation of the peaks is of the order of a few nanometers and therefore is in the range of peak separation reported by Hillman et al. ${ }^{16}$ and Lawandy et $a l .{ }^{17}$ in their studies of bichromatic emission from $\mathrm{cw}$ dye lasers.

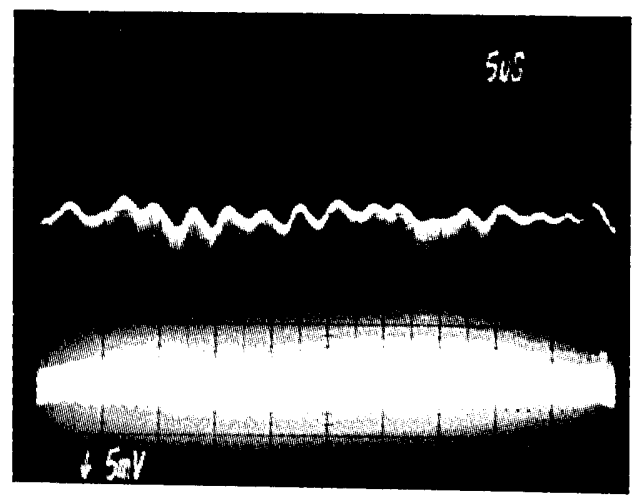

(a)

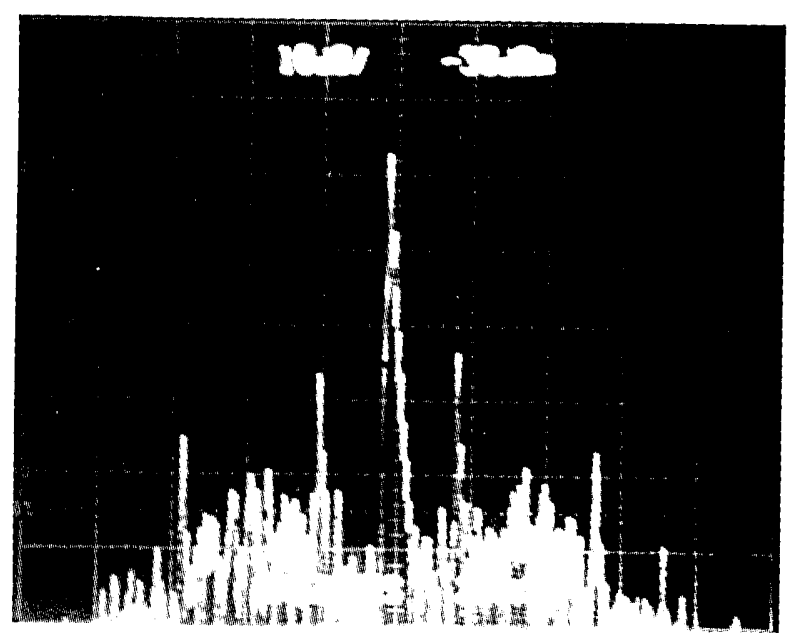

(b)

Fig. 5. Irregular pulse-train envelope at $L=L_{\mathrm{opt}}-60 \mu \mathrm{m}$ and associated homodyne spectrum. Instrument settings are the same as in Fig. 2.
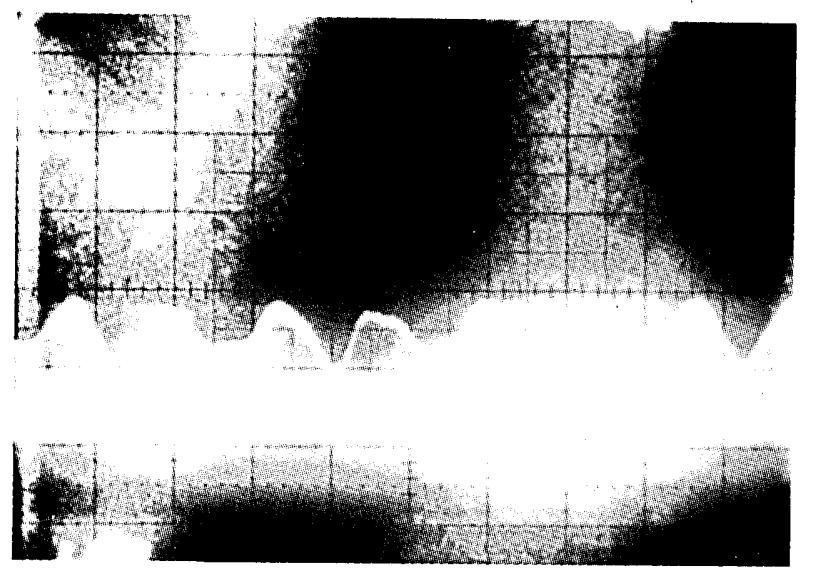

(a)

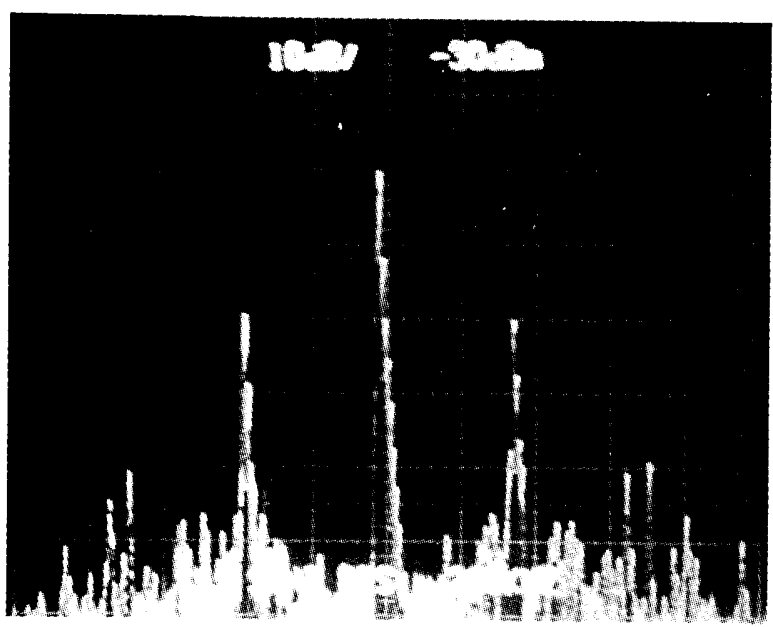

(b)

Fig. 6. Irregular modulation of mode-locked pulse train at $L=L_{\text {opt }}$ $-123 \mu \mathrm{m}$. Instrument settings are the same as in Fig. 2.

\section{DISCUSSION}

The effects reported here represent several different aspects of SPML operation. It would be fair to expect that the pulse-train instabilities described here would arise from the nonlinear dynamics of short pulse formation where models that provide good agreement with pulse-shape data have been derived without wavelength considerations. ${ }^{18}$ Furthermore, the research of New and Catherall suggests that, because of spontaneous emission, the pulse train for a SPML laser is inherently unstable in certain length regimes. ${ }^{8,9}$ More heuristic has been the work of Ogawa and Hamamura, who studied the chaos predicted by the Maxwell-Bloch equations under sinusoidal driving. ${ }^{19}$ Of interest in a specific model of our SPML work would be the keystone role of the linear filter element in the formation of the erratic envelope, for without the filter the pulse train is stable. That the relevant characteristic of the filter is its finite bandwidth (response time) is supported by the observation that the character and magnitude of the erratic envelope are independent of filter detuning but the effect increases for filters of decreasing bandwidths. 


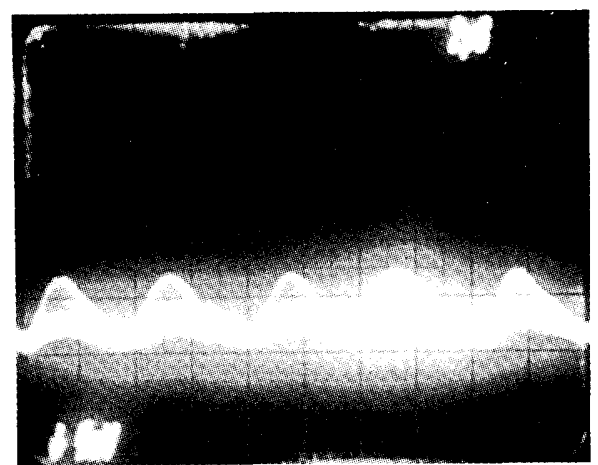

(a)

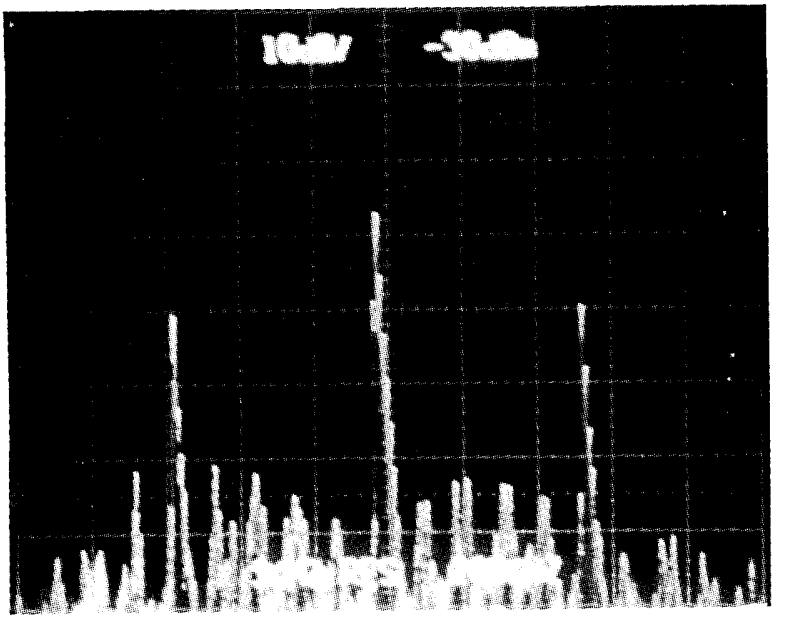

(b)

Fig. 7. Modulation of pulse train at $L=L_{\mathrm{opt}}-128 \mu \mathrm{m}$. The lowfrequency components in the homodyne spectrum of (b) suggest that a period -5 bifurcation has occurred since Fig. 6(b). Instrument settings are the same as in Fig. 2.

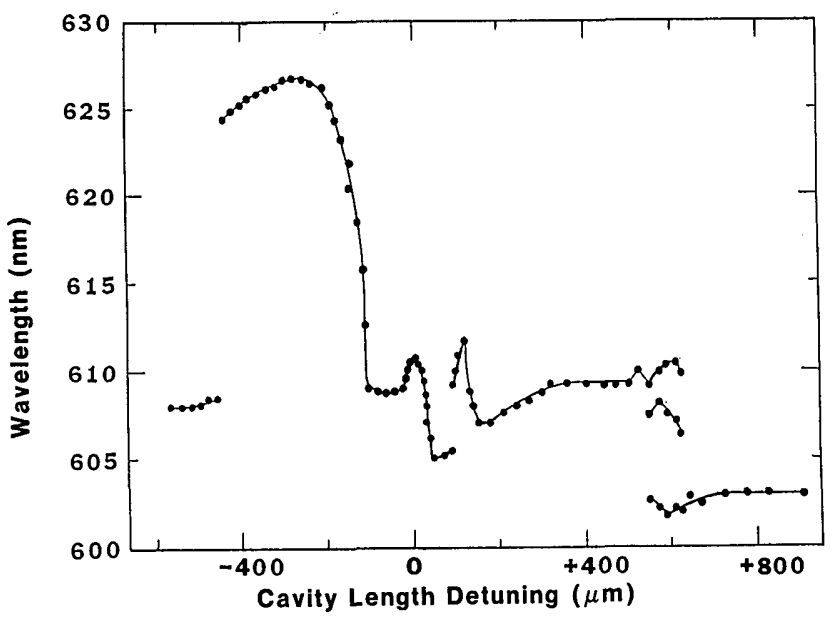

Fig. 8. Peak lasing wavelength as a function of cavity detuning for a free-running SPML dye laser. Typical error bars are less than the spot diameter.
To contrast with the filter-dependent erratic envelope in the region about $L_{\text {opt }}$ is the periodic modulation depicted in Fig. 3 that occurs for long cavities whether the filter is present or not. Associated with this effect is the linear shift of the modulation frequency with cavity-length increase, as shown in Fig. 4. Importantly, the threshold detuning value of $+92 \mu \mathrm{m}$ and the best-fit slope of $1.09 \mathrm{kHz} / \mu \mathrm{m}$ are independent of the filter. Associated with this transition is the abrupt change in wavelength (see Fig. 8) and the doublehumped emission of Fig. 9(c) that characterize the critical detuning of $+92 \mu \mathrm{m}$. This marked transition in the emission spectra of the dye laser at the onset of the periodic amplitude modulation of the pulse train suggests that wavelength and band considerations are also important in the dynamic model. To clarify these issues, more work is needed on the effects of bandwidth-limiting tuning filters on the output of SPML lasers.

In the absence of a rigorous theoretical model, we may speculate on plausible explanations for the shifts in wavelength with cavity detuning. One possibility would be that the shifts arise from the dispersion of the Rhodamine dye jet. Given that the gain from the argon laser arrives periodically with a fixed frequency $\nu_{\mathrm{Ar}}$, any stable pulse propagating in the cavity has to satisfy the condition

$$
k \nu_{\mathrm{Ar}}=c / 2 n(\lambda) L,
$$

where $c$ is the speed of light, $n$ is the effective wavelengthdependent index of the cavity, $L$ is the cavity length, and $k$ is an integer. The propagating pulse can then fulfill condition (1) if it changes its wavelength to keep its effective cavity length constant. Because most of the cavity is free space, $n(\lambda)=n_{0}(1-l / L)+(l / L) n_{d}(\lambda)$, and Eq. (1) may be rewritten as

$$
(l / L) n_{d}(\lambda)=c / 2 L \nu_{\mathrm{Ar}}-n_{0}(1-l / L) .
$$

Here, $n_{0}$ is the index of free space, $n_{d}(\lambda)$ is the dispersive index of the dye, $l$ is the length of the jet, and, in accordance with our setup, $k=1$. However, because the dispersion of the dye is scaled by $l / L \approx 10^{-6}$, this explanation cannot account for the 25-nm variation in $\lambda$. In other words, because the dye jet is only approximately $200 \mu \mathrm{m}$ long, its index must about double to compensate for a similar shift in cavity length.

More fundamentally, it is the group velocity,

$$
v_{g}=c /(n+\nu \mathrm{d} n / \mathrm{d} \nu),
$$

and not the phase velocity, $c / n$, that we need to consider in Eq. (1) above. In Eq. (3), $\nu \mathrm{d} n / \mathrm{d} \nu$ is the dispersion, which, for a homogeneous laser operating near line center, is

$$
\nu \mathrm{d} n / \mathrm{d} \nu=\operatorname{cg}(\nu) /\left(2 \pi \Delta \nu_{h}\right) .
$$

Here, $g(\nu)$ is the small-signal gain and $\Delta \nu_{h}$ is the homogeneous linewidth. For our laser $\nu(\mathrm{d} n / \mathrm{d} \nu)$ may be appreciable-of the order of 0.4-0.8. Consequently a variation in group velocity might contribute to the wavelength variations observed here.

Alternatively, a simple band model argument can also lead to wavelength shifts. The upper lasing state $S_{1}$ of the dye is actually a $30-\mathrm{nm}$ band of vibrational sublevels with an intraband relaxation time of the order of a few picoseconds. ${ }^{18}$ 
Because the $S_{1}-S_{0}$ transition time is approximately $5 \mathrm{nsec}$, the excited dye molecules typically accumulate at the bottom of the band, and it is a good approximation, under normal lasing conditions, to ignore the band structure altogether. Nonetheless, in a synchronously pumped configuration, a shortening of the cavity could cause the pulse to arrive before the excited molecules have cascaded to the bottom of the $S_{1}$ band. This blue shifts the lasing wavelength. Given a $30-\mathrm{nm}$ bandwidth and an intraband relaxation time of 4 psec, this picture predicts roughly a 5 -nm shift in wavelength for a $100-\mu \mathrm{m}$ cavity detuning. This figure is close to the slope at several places of the wavelength detuning curve of Fig. 8. Of note here is the recent study by Hong and Haken $^{20}$ that suggests that the band structure of dye molecules may account for the spectral behavior observed in Refs. 16 and 17.

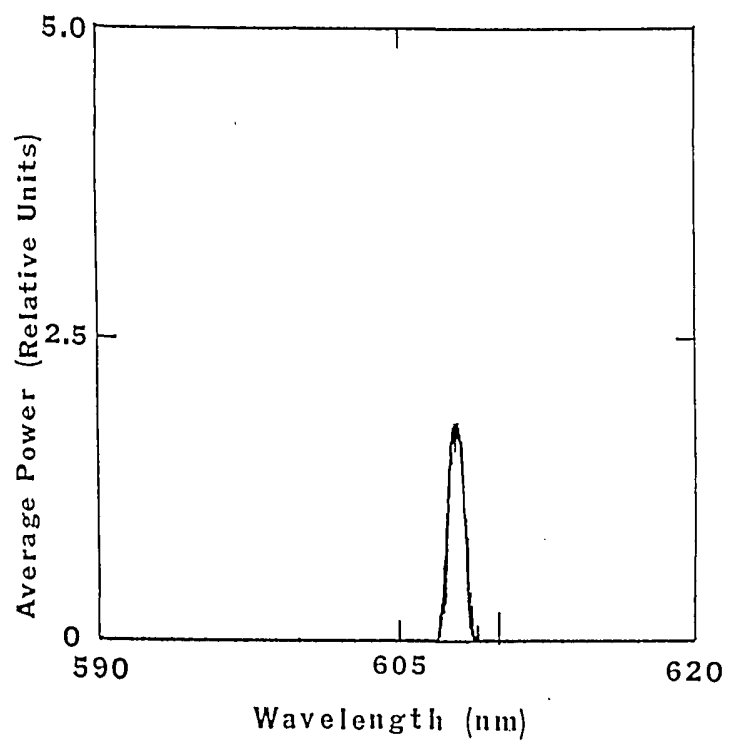

(a)

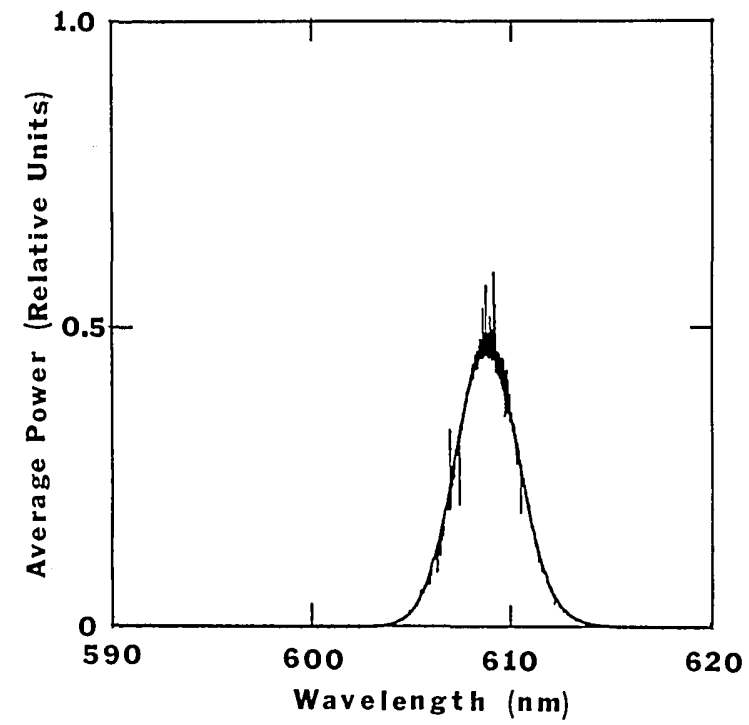

(b)
More experimental work needs to be done on the multipeaked emission spectra. It is tempting to associate our data with the bichromatic emission observed by Hillman $e t$ $a l .{ }^{16}$ and Lawandy et al., ${ }^{17}$ but the dramatically different experimental configurations and our relatively broad, nondistinct spectral peaks are significant obstacles to such an association. The 40-psec pulse $(1.2 \mathrm{~cm})$ and our cavity geometry make our laser essentially unidirectional in the pulse-active medium interaction. Thus no pulse-shortening, spectral splitting dispersion grating can be set up.

Although the effects reported here are pertinent characteristics of a synchronously pumped mode-locked system, it is not anticipated that these effects will be easy to reproduce with a single simple theoretical model. Existing models for SPML lasers are already highly complex, and, in most cases, they omit the nonperiodic behavior, bandwidth-limiting fil-

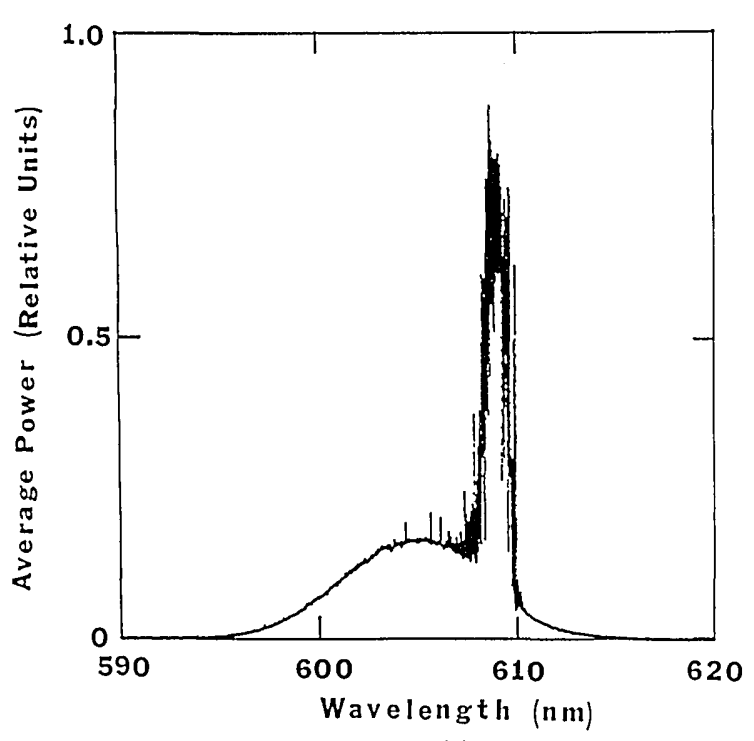

(c)

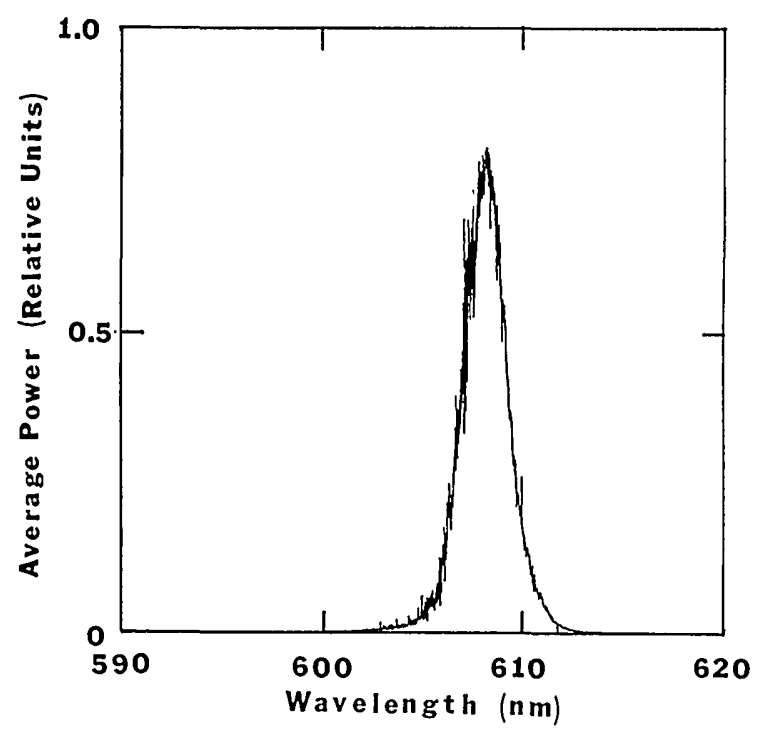

(d)

Fig. 9. Emission spectra of a free-running SPML dye laser for (a) $L=L_{\mathrm{opt}}-35 \mu \mathrm{m}$, (b) $L=L_{\mathrm{opt}}$, (c) $L=L_{\mathrm{opt}}+92 \mu \mathrm{m}$, and (d) $L=L_{\mathrm{opt}}+280$ $\mu \mathrm{m}$. The horizontal scales are identical; however, the vertical scale of (a) is, as marked, five times less than those of $(\mathrm{b})-(\mathrm{d})$. 


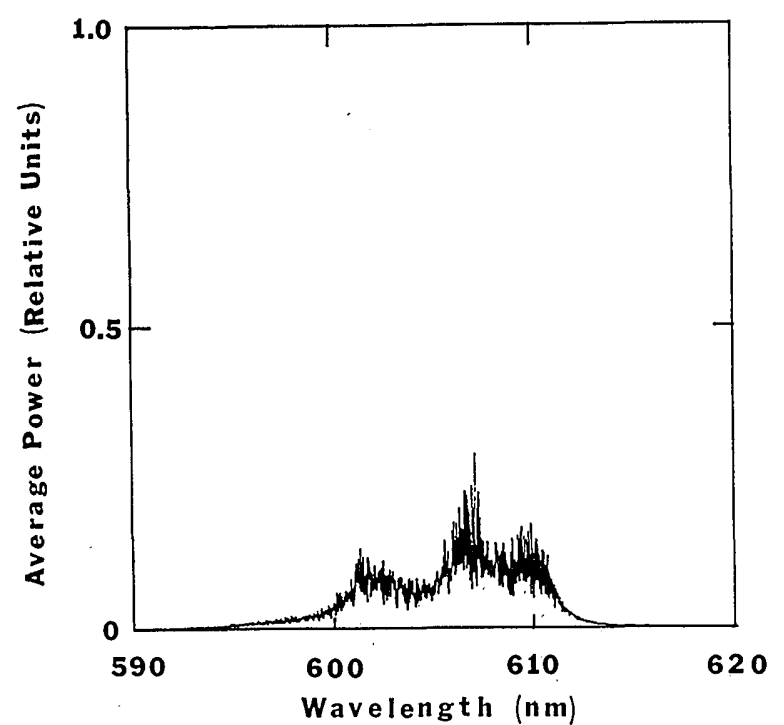

Fig. 10. Triple-peaked emission spectrum of a free-running SPML dye laser at $L=L_{\text {opt }}+585 \mu \mathrm{m}$.

ters, and dye spectral characteristics that are apparently fundamental to these experimental observations.

\section{ACKNOWLEDGMENTS}

This research was supported in part by the National Science Foundation and by Tektronix Inc. D. L. MacFarlane wishes to thank Bahram Zandi for his kind instruction in the operation of the laser system.

\section{REFERENCES}

1. L. E. Hargrove, R. L. Fork, and M. A. Pollack, "Locking of HeNe laser modes by synchronous intracavity modulation," Appl. Phys. Lett. 5, 4-5 (1964).

2. P. M. W. French, A. S. L. Gomes, A. S. Gouveia-Neto, and J. R. Taylor, "Spectral optimisation of a synchronously mode-locked femtosecond dye laser," Opt. Commun. 60, 389-392 (1986).

3. D. J. Bradley and A. J. F. Durrant, "Generation of ultrashort dye laser pulses by mode locking," Phys. Lett. A 27, 73-74 (1968).

4. C. K. Chan and S. O. Sari, "Tunable dye laser pulse converter for production of picosecond pulses," Appl. Phys. Lett. 25, 403406 (1974).
5. Z. A. Yasa and O. Teschke, "Picosecond pulse generation in synchronously pumped dye lasers," Opt. Commun. 15, 169-172 (1975).

6. A. Scavennec, "Mismatch effects in synchronous pumping of the continuously operated mode-locked dye laser," Opt. Commun. 17, 14-17 (1976).

7. J. Herrmann and U. Motschmann, "Formation of synchronously mode-locked dye laser pulses," Opt. Commun. 40, 379-384 (1982).

8. G. H. C. New and J. M. Catherall, "Problems in the self-consistent profile approach to the theory of laser mode-locking," Opt. Commun. 50, 111-116 (1984).

9. G. H. C. New and J. M. Catherall, "Perturbations and instabilities in laser mode-locking dynamics," in Optical Instabilities, R. W. Boyd, M. G. Raymer, and L. M. Narducci, eds. (Cambridge U. Press, Cambridge, 1986), pp. 196-211.

10. B. Xiaoyi and W. Cunkai, "Stability criteria for pulse solution of a synchronously pumped mode-locked dye laser," Opt. Lett. 12, 251-253 (1987).

11. J. Kluge, D. Wiechert, and D. Von der Linde, "Fluctuations in synchronously mode-locked dye lasers," Opt. Commun. 51, 271277, (1984).

12. S. L. Shapiro, R. R. Cavanagh, and J. C. Stephenson, "Streakcamera observations of the pulse emission from a synchronously pumped continuous-wave mode-locked dye laser," Opt. Lett. 6, 470-472 (1981).

13. T. M. Baer and D. D. Smith, "Noise in picosecond laser systems: Actively mode locked $\mathrm{cw} \mathrm{Nd}{ }^{3+}: \mathrm{YAG}$ and $\mathrm{Ar}^{+}$lasers synchronously pumping dye lasers," in Ultrafast Phenomena IV, D. H. Auston and K. B. Eisenthal, eds. (Springer Verlag, Berlin, 1984), pp. 96-98.

14. J. P. Zheng, U. Sen, D. M. Benenson, and H. S. Kwok, "Observation of periodicity multiplication in a synchronously pumped dye laser," Opt. Lett. 11, 632-634 (1986).

15. D. L. MacFarlane and L. W. Casperson, "Pulse-train instabilities in a mode-locked argon laser," J. Opt. Soc. Am. B 4, 17771780 (1987).

16. L. W. Hillman, J. Krasinski, R. W. Boyd, and C. R. Stroud, Jr., "Observation of higher order dynamical states of a homogeneously broadened laser," Phys. Rev. Lett. 52, 1605-1608 (1984).

17. N. M. Lawandy, R. Sohrab Afzal, and W. S. Rabinovich, "Bichromatic emission in a ring dye laser," Phys. Rev. A 36, 17591770 (1987).

18. L. W. Casperson, "Coherence effects in synchronously pumped mode-locked dye lasers," J. Appl. Phys. 54, 2198-2208 (1983).

19. T. Ogawa and E. Hamamura, "Dynamical properties of the multimode laser with modulated inversion," Opt. Commun. 61, 49-54 (1987).

20. Hong Fu and H. Haken, "Semiclassical dye equations and the unidirectional single-frequency operation," Phys. Rev. A 36, 4802-4816 (1987).

(see overleaf) 


\section{L. MacFarlane}

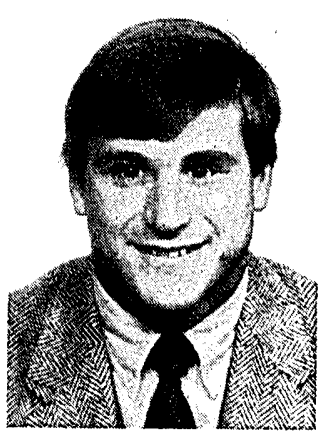

Born in Morristown, New Jersey, on November 13, 1962, D. L. MacFarlane received the B.S. degree in 1984 and the M.S. degree in 1985, each in electrical engineering and each from Brown University, Providence, Rhode Island. Currently he is working toward his Ph.D. at Portland State University in Portland, Oregon. His research interests tend toward laser mode locking and optical instabilities. He is a member of Sigma Xi.
A. A. Tovar

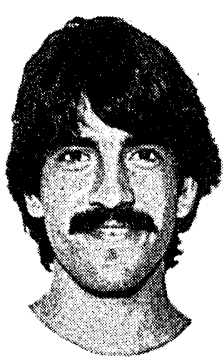

A. A. Tovar was born in Portland, Oregon, in 1964. He received B.S. degrees in both electrical engineering and mathematics in 1987 from Portland State University, Portland, Oregon, where he is now pursuing an M.S. degree in applied optics. His current research activities are in tapered graded index fibers and nonlinear dynamics. 